\title{
Estimation of the $\mathrm{X} / \gamma$ - ray shielding properties of different glass system using Monte Carlo Simulation
}

\author{
Ayano Shanko, MD ${ }^{a}$, Ajay Prakash Pasupulla ${ }^{\mathrm{b}}$, Paulos Ashebo Dojamo ${ }^{\mathrm{c}}$, Firos. A , and Dr. Sandeep \\ Gupta $^{\mathrm{e}}$
}

Assistant Professor, Internal Medicine, College of Medicine and Health Sciences, Nigist Eleni Mohammed Memorial Comprehensive Specialized Hospital, Wachemo University, Hosanna, Ethiopia.

B

Professor, Oral Pathology, Department of Dentistry, College of Medicine and Health Sciences, Nigist Eleni Mohammed Memorial Comprehensive Specialized Hospital, Wachemo University, Hosanna, Ethiopia.

Lecturer, Department of Dentistry, College of Medicine and Health Sciences,

Nigist Eleni Mohammed Memorial Comprehensive Specialized Hospital,

Wachemo University, Hosanna, Ethiopia.

Assistant Professor, Department of Computer Sciecne and Engineering, Rajiv

Gandhi University, Doimukh, Arunachal Pradesh.

E

Assistant Professor, Department of Electrical Engineering, JECRC University, Jaipur, Rajasthan 303905, INDIA

Corresponding Author Email: shanko.ayano@yahoo.com

Article History: Received: 11 January 2021; Accepted: 27 February 2021; Published online: 5 April 2021

\begin{abstract}
The aim of the research is to estimate the X-ray shielding properties of different glass systems using Monte Carlo Simulation. X-ray glass is also known as radiation shielding glass. Glass provides protection against the absorption of energy radiation. The shielding layer is formed by a high concentration of lead and barium. The mass attenuation coefficient, the effective atomic number and the effective electron density are used to determine the position of gamma-ray photons in matter. Shield characterization in terms of mass attenuation coefficient $(\mu \mathrm{m})$, transmission fraction $(\mathrm{T})$, effective atomic numbers (Zeff), half-value layer (HVL) and exposure build-up. factor (EBF) of a glass system is estimated by the Monte Carlo Simulation. The random sampling and statistical analysis are computed using the monte carlo simulation. Various external factors are considered as the input parameters. The different composition of the glass will be examined using the Monte Carlo simulation and the shielding capability would be determined for the various samples.
\end{abstract}

Keywords: Monte Carlo Simulation, Shielding Properties, Radiation, Mass attenuation coefficient, Half value layer (HVL), Exposure build-up factor (EBF).

\section{Introduction}

Higher-energy photon interactions are very important in radiation medicine, biology, nuclear engineering. Transparent to visible light and absorbing gamma rays and neutrons are the double function of the glass and provide a shielding effect (Savage 1972). The shielding effect is taken as an important part of the long-term storage of radioactive waste product. Mass attenuation coefficient, partial interaction and effective atomic number theoretical calculation are included on the basis of the glass material used. The highest total attenuation coefficient is found in $\mathrm{PbO}$-based silicate glass, followed by phosphate and borate glasses. In each of the glasses. In each of the glass matrices the Compton scattering interaction has been found.

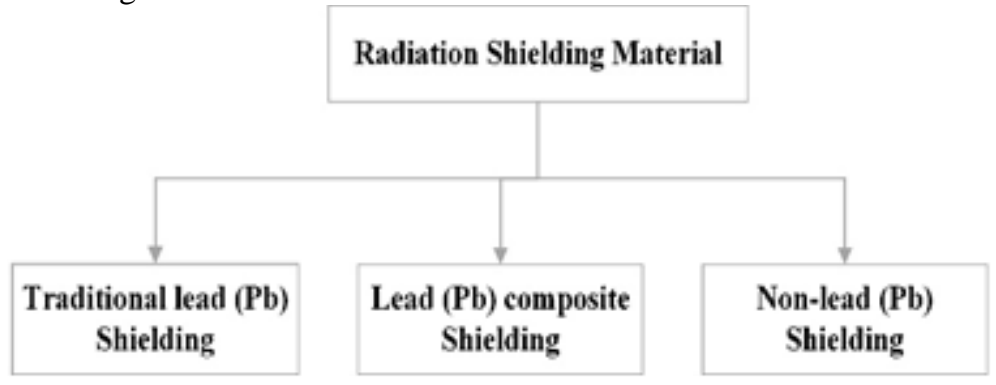

Fig.1 Types of shielding Material 
Variety of radiologic application uses the radiation shielding material. The uses of radiation in diagnosing and treating patients has been used in the medical institution form saving the lives. The tree major principles of mitigating external radiation exposure are shielding. The effective way to reduce the radiation exposure is using certain absorbing material such as Plexiglas (Kaur et al. 2019) for beta particles and lead for X-rays and gamma rays. This paper investigates the estimation about the $\mathrm{X}$ - rays and the gamma rays. The Fig. 1 illustrate the type of radiation shielding material used. The radiation shielding material have been manufactured from lead $(\mathrm{Pb})$. In variety of application the Lead shielding material is most commonly used. This is mainly used in the diagnostic imaging, radiation therapy, nuclear and industrial shielding. This mainly focused on the $\mathrm{X}$ ray attenuating garments.

The radiation shielding material is divided into three types they are traditional $(\mathrm{Pb})$ shielding, Lead $(\mathrm{Pb})$ composite shielding and the Non lead $(\mathrm{Pb})$ shielding. The traditional lead shielding is purely made of the lead chemical element. It is useful shield against X-ray and gamma- ray radiation due to its high density of lead. The lead cannot be worn as garb in its pure form. To make it as flexible lead its blended and mixed with the other addictive. In traditional lead radiation shielding material there are typically three standard level of protection. The lead $(\mathrm{Pb})$ composite shielding is the mixture of lead and other light weight metal (Limkitjaroenporn et al. 2011). They are developed by their own properties the composite blend nature varies. They are very light and composite. The protection level is same as the lead. The non- lead shielding is the last type of the radiation shielding material. They are similar to the properties of the composite lead shielding effect. The non-lead shielding material is blended with the heavy metals. They include tin $(\mathrm{Sn})$, antimony $(\mathrm{Sb})$ and other elements. This provides very ow level protection. The three shielding material have unique properties, benefits and features.

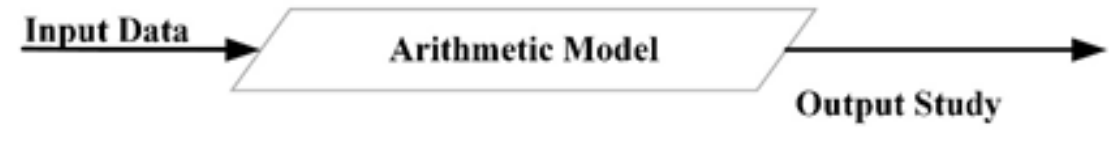

Fig. 2 Arithmetic Model

The random sampling and statistical analysis are computed in the Monte Carlo Simulation method. 'So called what if analysis' this could determine the Monte Carlo simulation (Dong et al. 2019) A large number of input parameter is included in this model. When the input is processed it give one or more outputs. The Fig.2 represent the arithmetic model of Monte Carlo simulation. Based on the various external factor the input parameter of the model depends. Due to the systematic variation of the input parameter and the factors the system is subjected to risk. Base case is defined as the variation in the deterministic model. Based on the risk associated with the various input parameter the effective model must be considered.

The medical imaging and the radiation therapy system mostly use the gamma and the $\mathrm{X}$ radiation. In the distribution of photon flux in every object the buildup factor is considered to be the important factor. In calculation of radiation dose, the photon buildup factor is considered as the important one. Using the Monte Carlo simulation, it is observed that the buildup factor of gamma and X-ray photons in the energy range $0.2-2 \mathrm{MeV}$ in water and the soft tissue. They are compared with the existing buildup factors. The researchers had shown great interest in developing better glass system for new technological application. For the photonic application the glass material is considered as the promising material. For avoiding the leakage of reactant gases which implicate the series of thermal, mechanical and chemical requirements the sealing material is used to provide the tightness. To satisfy the requirement the glass ceramic material offers the best views.

Based on the data we analysis the X-ray and the gamma ray shielding properties of different glass system using the Monte Carlo Simulation. The different shielding materials were studied and discussed; the use of the Monte Carlo simulation method has been studied using the larger number of input data analysis. The feasibility study of the shielding system for the use of a different application of safety from radiation is mentioned and the improvement is made to estimate the shielding properties.

The section 2 defines the literature review of the glass system, radiation material and the glass properties of different method. Section 3 describe the problem statement and the improvement to be made in the future work. Section 4 defines the proposed system, the idea and the improvement in the system is defined in this section. The section 5 represents the result and discussion part and the section 6 defines the conclusion.

\section{Related Work}

(Wang and Modest 2006) proposed the method known as the Photon Monte Carlo Simulation for radiative transfer in gaseous media represented by discrete particle fields. This method is about the evaluation of the heating problem using the Monte Carlo simulation method. this deals with the photon ray model and the spatial location with the $2 \mathrm{D}$ particle. In this method the particle position vector about the geometric information is only known by the system. the implementation of the Monte Carlo simulation focusses on the emission from the particle. A bundle of photons is emitted from the field. The optical thickness is very much important in the carlo simulation method. Self-absorption is used if the optical is very thin. This helps in the interaction between the point masses the cone approaches, the optimal opening angle and more efficient number of particles per cell is found. The disadvantage of this technique is it consume more time. The time consumption may lead the active photon to cause any defects.

The optimization of glass-ceramic sealant composition in the $\mathrm{MgO}-\mathrm{BaO}-\mathrm{SiO} 2$ is proposed by (Pascual, Guillet, and Durán 2007). This states the glass ceramic composition and the thermal expansion coefficient of the system. 1118 
The major glass system used in this method is $\mathrm{MgO}-\mathrm{BaO}-\mathrm{SiO} 2$. The suitable properties of the glass system were notes and then the glass material is melted in the alcohol. The laser light dispersion method is used to estimate the glass particle proportion. The glasses without zinc are observed in this method and the crystalline of barium Zinc silicate is promoted in the zinc containing glass. The expected decrease of viscosity is produced and the this leads to the greater wettability of the glass to the steal. The better bonding in relation to the glass is made. The disadvantage of the system is it need high heating capacity and the different methods are involved to melt and to estimate the composition of the glass particle.

(Akkurt et al. 2010) proposed the method of gamma-ray shielding properties of concrete including barite at different energies. The paper investigates the radiation shielding properties of barite and concrete produced with barite. The standard shielding material of lead is compared with the result obtained in the system. The linear attenuation coefficient of barite is analyzed in this method. The cement material is used in this method, the ratio of the cement material is noted. The linear attenuation of the concrete is measured using the gamma spectrometer. The method is about the shielding properties of the gamma rays. The concrete has hard particle and the transmission rate of the gamma ray is large as the concrete allows the particle to easily pass through. The thickness of the barite is low when compared to the lead. This is major disadvantage if the Gamma shielding rays.

Utilization of recycled cathode ray tubes glass in cement mortar for $\mathrm{X}$ ray radiation- shielding application is about the recycled glass derived from the cathode ray tube (Pascual, Guillet, and Durán 2007). This is suitable for preparing cement mortars for X-ray radiation-shielding application. In this method various properties were involved to produce the recycled glass. The X-ray radiation properties and the management of cathode ray are complicated to perform. The shielding effect is weak due to the transfer of the large number of particles. The increase in the glass content could gradually increase the compressive strength. This system is mostly used for the building application. The disadvantage of the system is high density and the low radiation performance.

The gamma ray shielding properties of $\mathrm{TeO} 2-\mathrm{ZnF} 2-\mathrm{As} 2 \mathrm{O} 3-\mathrm{Sm} 2 \mathrm{O} 3$ glasses proposed by (Gaikwad et al. 2018) defines the working condition of the series of glass. This is done by the quenching method. This is done by the narrow beam geometry method. This is widely used in the measurement of the linear attenuation coefficient. The energy of the glass system ranges from $0.123-1.33 \mathrm{MeV}$. This determines the shielding effect with the titanium and the silicon material. The attenuation coefficient is very low when compared to the lead. This shielding capability of the glass sample against the gamma rays are very low.

\section{Problem Statement}

The main problem in glass shielding is heating process. The heat is differently observed by the various particle. The lead X-ray primarily used in the medical purposes but the radiation effect is very large and this may also cause some defects to the patient. The existing method the cone clay simulation consumes more time than the other method. The protection level of the gamma rays and the X- rays are very low in the existing method. The calculation of mass attenuation, effective atomic number, the half value layer is not clearly determined in the existing method. the characterization of different properties of the glass material is not defined clearly. The optimization of the glass system combination element was not defined in a proper manner. The capabilities of the Monte Carlo simulation application are not defined. So, to overcome this disadvantage the improvement are made in the Monte Carlo Simulation to estimate the X-ray and gamma rays shielding properties of different glass system using Monte Carlo Simulation.

\section{Proposed system}

The estimation of the X-ray and the gamma- rays are computed using the Monte Carlo simulation method. The glass material with different properties were taken and subjected to the estimation. The studding capabilities of the Monte Carlo simulation in the application of shielding material could determine the factors of the glass material. In the Monte Carlo simulation, the large number of input data can be given and one or more output data is analyzed. The shielding properties of the glass depends on the material used in the radiation shielding. By measuring the density of glass and calculating the molar volume and the oxygen packing density the physical properties of the glass material are calculated. In this method the Monte Carlo simulation, GEANT4 model is used. the glass material used in the estimation is melted in the alcohol for 1-3 hour. This is carried out by the laser light dispersion method. Then the material is analyzed and the various properties of the X-ray and the gamma- rays are represented. The optimization of glass system of the combination of the element is carried out. Thus, the different combination of shielding material based on the glass system is examined and the properties are determined.

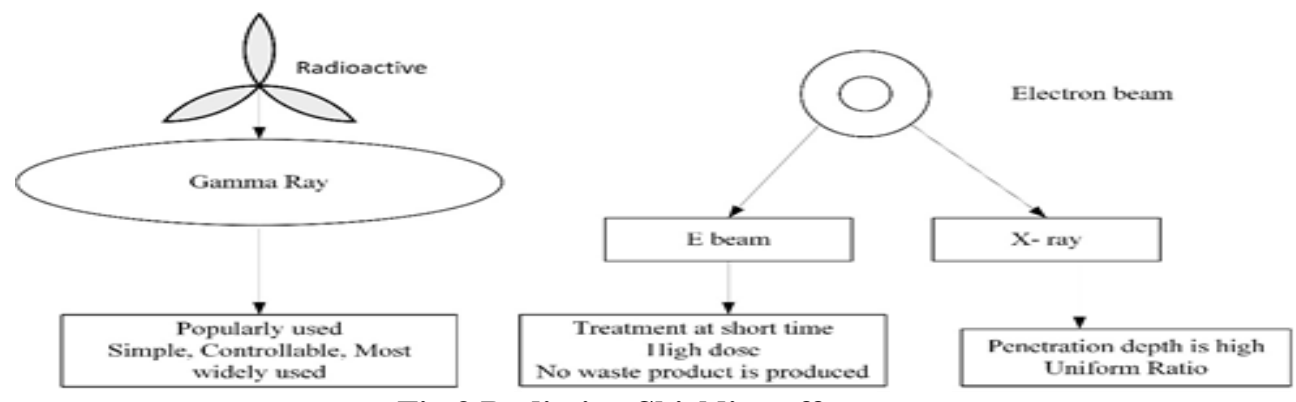

Fig.3 Radiation Shielding effect 
The Fig.3 illustrate the radiation shielding effect. The radioactive substance give rise to the gamma rise and the electron give rise to the $\mathrm{E}$ beam and the $\mathrm{X}$-ray. The shielding properties of the radiation and the properties are mentioned. The radioactive substance is giving rise to the gamma ray. The gamma rays are popularly used and it is simple to recognize, it is easy to control and its widely used. The electron substance is divided into e beam and the X-rays the e beam are high dose particle so it causes a large effect in the during the radiation of shielding particle. The $\mathrm{X}$ - rays have high penetration rate and it easily pass through the glass material. The uniform ratio is distributed among the large area. The X-ray and the gamma ray shielding properties are mentioned below.

\section{1 $\mathrm{X}$ - ray radiation shielding properties}

The electromagnetic radiation is termed as the X-rays. They are the powerful waves of the electromagnetic energy. The wavelength of the X-ray ranging from 0.01 to 10 nanometer with respect to the frequency range. The motion of picture is used in the study of human physiology in the medical science. There are two types of X-rays available. They are hard ray and the soft ray. The hard X-ray is produced when the photon energies and the soft X-rays are produced when the photon energy is very low. The hard rays are used inside the object due to their penetrating ability. The X- ray crystallography is used in determining the crystalline structure of the X-rays. In the X-ray shielding laboratory the X-ray shielding test is performed. Its is implemented in the medical diagnostic examination. The medium frequency $\mathrm{X}$ - ray unit is installed in the laboratory. Below the $\mathrm{X}$-ray tube head the collimators with double layer of lead plate is place and the beam is allowed to pass through. Based on the projected distance the angle is calculated. Finally, the measured rays are subjected to the Monte Carlo simulation method and then the X-rays properties for different glass is estimated

\subsection{Gamma- ray shielding properties.}

The penetrating form of the electromagnetic radiation is termed as the gamma ray. This consist of the shortest wavelength and highest photon energy. Radioactive decay is formed by the gamma rays from the few kiloelectronvolt. They are produced by number of astronomical process, in this high energy electrons are produced. The gamma source is defined as the sample of gamma ray-emitting material used for irradiating or imaging purpose. This is also known as the radioactive source. To prevent the radioactive contamination and transported in heavy shielding the gamma source is usually used. The subjected rays are measured and then passed to the Monte Carlo simulation and the output is obtained.

\section{Result and Discussion}

The attenuation of the photon in the matters obey the attenuation rule. The following equation is generally considered for the gamma and $\mathrm{X}$ - ray photons.

$$
I=B(F, y) I_{o} \exp ^{-\beta y}
$$

In this the $\mathrm{B}$ denotes the buildup factors due to the effect of the photon. $\mathrm{F}$ denotes the photon energy and the $\beta y$ denotes the penetration depth. The value of the buildup factor is assumed to be unity. The atomic shielding property of the material is defined as $\sigma \mathrm{v}$ and $\sigma \mathrm{d}$. The following expression gives the buildup factor.

$$
\begin{gathered}
B=A e^{-\alpha \mu r}+(1-A) e^{-\beta \mu r} \\
B=1+a \mu r e^{b \mu r}
\end{gathered}
$$

As mentioned above the limit condition is satisfied by $\mathrm{B}$;

$$
\begin{array}{r}
\mathrm{B}=1 \text { at } \sum_{v}=\infty \\
\mathrm{B}=\infty \text { at } \sum_{v}=\infty
\end{array}
$$

The above equation determines the attenuation factor of the $\mathrm{x}$ ray and the gamma rays.

From the measurement analysis the error or linear attenuation is fund to be $5 \%$. The mass attenuation coefficient is compared with the linear attenuation coefficient which is obtained by using the Monte Carlo Simulation method.

\section{Mass Attenuation Coefficient}

The energy range of the function photon with the variation of prepared glasses as the function of the photon energy $(\mu \mathrm{m})$ is measured. The present glass experimental data is given as $0.125,0.66,1.178,1.272$ and 1.44 $\mathrm{MeV}$ are calculated using the Monte Carlo Simulation and shown in the graph. The Fig.4 represent the attenuation variation.

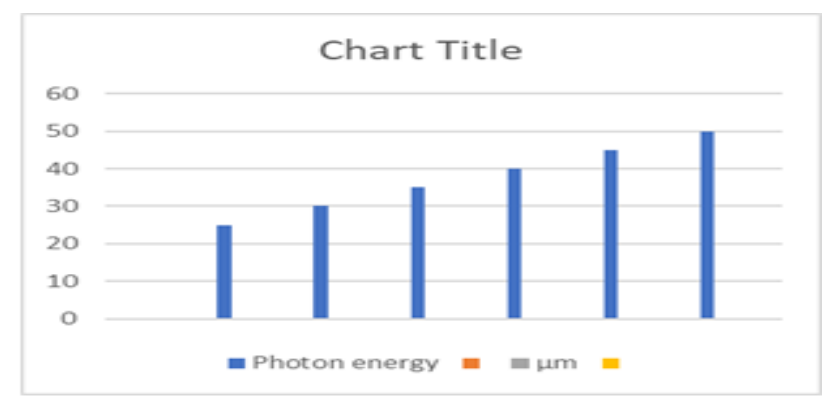

Fig.4 Variation of mass Attenuation Coefficient 
From the above result it observed that the incident photon depends on the $\mu \mathrm{m}$. The prepared glass could have partial photon interaction process. The low energy level the $\mu \mathrm{m}$ values of the glass get decrease and the photon energy level get increase. Overall calculation shows that lead value is very large.

Half value layer (HVL) and Mean free path (MFP)

The HVL is used to describe the effectiveness of the radiation shielding. The glass sample has more thickness, by using its initial intensity it removes the half the intensity photons. The superior radiation shielding matters are provide by very low HVL and MFP. For the prepared glass the higher shielding effect of gamma ray's effectiveness is experimented and the HVL values of the prepared glasses is measured.

Effective atomic number (Zeff)

The glass effectiveness increases with the increase in the lead content and it decrease with the increase in the photon energy. The photon energy ranges from 0.123 to $1.33 \mathrm{MeV}$. The slight variation in the effective atomic number could cause the variation in the glass sample which could result to the increased probability of the photon particle.

Exposure Buildup Factor (EBF)

The prepared glass EBF value range from $0.123-1.33 \mathrm{MeV}$. With the increase in the glass sample increase with the increase in the photon energy. this is due to the narrow beam transmission. The scattered photon has reached at the detector.

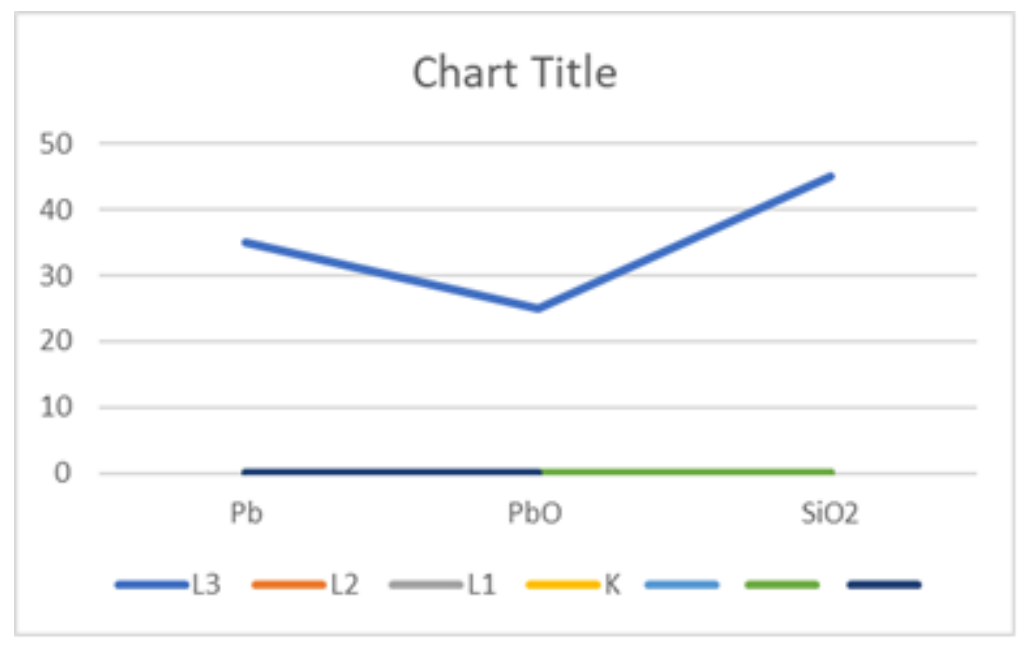

Fig.5 Exposure Buildup factor of the Lead

The Fig. 5 represent the exposure buildup factor of the lead material. The material like lead and the lead oxide were used.

\section{Discussion}

By the overall result it shows that the photon energy is most responsible for the glass shielding particle. The estimation of the $\mathrm{x}$ - ray and the gamma ray is calculated using the Monte Carlo simulation method and the analysis for the factors are determined. The mass attenuation factor, the effective atomic number, buildup factor and the exposure buildup factors are determined using this simulation. The Characteristic of the glass shielding properties are determined and the type of radiation material is discussed in the introduction part. Selection of the radiation material is important as they prevent the loss of attenuation. Thus, the estimation of the shielding properties are determined.

\section{Conclusion}

Gamma ray and X-ray attenuation coefficient, HVL, MFP. Zeff and EBF were calculated for the samples prepared. The observed result shows that gamma rays and X-rays are widely used in industries due to the higher shielding effect. The increase in attenuation is the result of an increase in photon energy. The lead content is identical to the mass attenuation coefficient due to the change in the effective atomic number and the electron density. Shielding properties protect the glass particles from the heating effect. The time consumption is reduced and the weakness in the glass particle are reduced. The increase in the photon level increases the strength of the glass particle. The radiation of the shielding effect is reduced. Overall, it shows that the $\mathrm{X}=$ ray and the gammarays are widely used in the radiation protection and the estimation of the X-ray and the Gamma ray particle are calculated, and the properties and the shielding characteristics are determined.

\section{Reference}

1. Akkurt, I, Hakan Akyildirim, B Mavi, S Kilincarslan, and C Basyigit. 2010. "Gamma-Ray Shielding Properties of Concrete Including Barite at Different Energies. ” Progress in Nuclear Energy 52 (7): 620 23.

2. Dong, M.G., O. Agar, H.O. Tekin, O. Kilicoglu, Kawa M. Kaky, and M.I. Sayyed. 2019. “A Comparative Study on Gamma Photon Shielding Features of Various Germanate Glass Systems. ” Composites Part B: Engineering 165 (May): 636-47. https://doi.org/10.1016/j.compositesb.2019.02.022. 
3. Gaikwad, D.K., M.I. Sayyed, Shamsan S. Obaid, Shams A.M. Issa, and P.P. Pawar. 2018. "Gamma Ray Shielding Properties of TeO2-ZnF2-As2O3-Sm2O3 Glasses.” Journal of Alloys and Compounds 765 (October): 451-58. https://doi.org/10.1016/j.jallcom.2018.06.240.

4. Kaur, Parminder, KJ Singh, Sonika Thakur, Prabhjot Singh, and BS Bajwa. 2019. "Investigation of Bismuth Borate Glass System Modified with Barium for Structural and Gamma-Ray Shielding Properties." Spectrochimica Acta Part A: Molecular and Biomolecular Spectroscopy 206: 367-77.

5. Limkitjaroenporn, $P, J$ Kaewkhao, P Limsuwan, and W Chewpraditkul. 2011. "Physical, Optical, Structural and Gamma-Ray Shielding Properties of Lead Sodium Borate Glasses." Journal of Physics and Chemistry of Solids 72 (4): 245-51.

6. Pascual, MJ, A Guillet, and A Durán. 2007. "Optimization of Glass-Ceramic Sealant Compositions in the System MgO-BaO-SiO2 for Solid Oxide Fuel Cells (SOFC). ” Journal of Power Sources 169 (1): 40 46.

7. Savage, J.A. 1972. "Glass Formation and D.S.C. Data in the Ge $\square$ Te and As $\square$ Te Memory Glass Systems." Journal of Non-Crystalline Solids 11 (2): 121-30. https://doi.org/10.1016/00223093(72)90294-3.

8. Wang, Anquan, and Michael F. Modest. 2006. "Photon Monte Carlo Simulation for Radiative Transfer in Gaseous Media Represented by Discrete Particle Fields. ” Journal of Heat Transfer 128 (10): 104149. https://doi.org/10.1115/1.2345431. 